\title{
EFFECT OF THERMAL RADIATION AND CHEMICAL REACTION ON MHD FLOW OF BLOOD IN STRETCHING PERMEABLE VESSEL
}

\author{
B. ZIGTA \\ WolaitaSodo University, College of Natural and Computational Science \\ Department of Mathematics \\ P.O. Box 138, ETHIOPIA \\ E-mail: tzigta@yahoo.com
}

\begin{abstract}
This paper focuses on the theoretical analysis of blood flow in the presence of thermal radiation and chemical reaction under the influence of time dependent magnetic field intensity. Unsteady non linear partial differential equations of blood flow consider time dependent stretching velocity, the energy equation also accounts time dependent temperature of vessel wall and the concentration equation includes the time dependent blood concentration. The governing non linear partial differential equations of motion, energy and concentration are converted into ordinary differential equations using similarity transformations solved numerically by applying ode45. The effect of physical parameters, viz., the permeability parameter, unsteadiness parameter, Prandtl number, Hartmann number, thermal radiation parameter, chemical reaction parameter and Schmidt number on flow variables, viz., velocity of blood flow in vessel, temperature and concentration of blood, has been analyzed and discussed graphically. From the simulation study the following important results are obtained: velocity of blood flow increases with the increment of both permeability and unsteadiness parameter. The temperature of blood increases at the vessel wall as the Prandtl number and Hartmann number increase. Concentration of blood decreases as time dependent chemical reaction parameter and Schmidt number increases.
\end{abstract}

Key words: stretching velocity, similarity transformations, time dependent magnetic field intensity, thermal radiation, chemical reaction.

\section{Introduction}

When blood flows through blood vessels it carries heat to different parts of our body. Heat can be transferred by conduction, convection and radiation. Radiation is a process which involves energy transfer by electromagnetic wave propagation which can occur in vacuum as well as in a medium. Experimental evidence indicates that radiant heat transfer is proportional to the fourth power of the absolute temperature, whereas conduction and convection are proportional to a linear temperature difference. Factors that affect quantity of thermal energy transfer in blood flow are: the thermal energy transfer coefficient of blood; density of blood; velocity of blood flow; the radius of the blood vessel; temperature of the tissues that surround the vessel.

Blood flows inside the arteries, veins and capillaries in one direction only. Blood is made up of plasma and cells. The plasma carries digested food, proteins, salt and waste products. In addition to the transport of materials, blood also serves in maintaining the water content of the body and distributing heat evenly throughout the body. Erythrocytes carry oxygen, white blood cells attack bacteria and the platelets help in blood clotting.

The Reynolds number is the ratio of the velocity, density and vessel radius to dynamic viscosity of blood; the quantity of heat carried by blood depends only on the Reynolds number and heat transfer coefficient of blood, and the temperature of the tissues surrounding the vessel. Magneto hydrodynamics is the study of electrically conducting fluid under the influence of the magnetic field. The magnetic fields with Newtonian and non-Newtonian fluids have wide applications in chemical engineering, bio-fluid mechanics and various industries. 
Thermal radiation effects in blood flow have applications in several medical research areas, viz., biomedical engineering, radiotherapy, MRI, CT scan, echocardiography and Doppler ultrasound. Infrared radiation can be applied directly in treatment of blood cancer by heat therapy to treat the infected areas of blood. Heat therapy is also used in the treatment of muscle pain, chronic wide spread pain and permanent shortening of muscles.

The effect of radiative heat transfer on blood flow in a stenosed artery was studied theoretically [1].The effect of temperature on blood flow in human breast tumor under laser irradiation using a numerical model was discussed in [2]. Blood flow with radiative heat transfer was discussed in [3]. Theoretical estimates of blood flow in arteries during the therapeutic procedure of electromagnetic hyperthermia used for cancer treatment were investigated in [4].

The field of study which relates the effect of applied magnetic field on biological fluids is called computational bio-fluid dynamics. This field of study helps to model and simulate blood flows in vascular passages such as cerebral and carotid arteries.

The flow of chemically reacting fluid mixtures have significant technological applications. Blood is a complex mixture of plasma, proteins, cells and other chemicals. An experimental study that depicts the metabolic activities in the presence of chemical reaction has been carried out [5]. An increment in plasma concentration of reactants occurs due to injection of drugs, hormones and metabolism. A theoretical model to study the impact of blood flow on the growth of thrombi by considering the interaction between different constituents of blood and chemical reaction has been developed in [6]. The effect of variable viscosity on chemically reacting magneto blood flow with heat and mass transfer has been studied in [7].

The effect of a porous medium on blood flow is significant in pathological study. A simulation study for blood flow through a contracting muscle, with a hierarchical structure of pores was presented in [8].The Brinkman equation helped to model the pathological blood flow when there is accumulation of fats in an arterial segment [9]. Flow of a Newtonian fluid in a porous medium in the presence of mass exchange between the constituents was analyzed in [10].

Erythrocytes are enriched with iron content of hemoglobin molecules so the blood flow will be influenced by the presence of an external field. Therefore, blood is an electrically conducting fluid in the presence of magnetic field. The blood flow exhibits magneto hydrodynamic properties. The steady MHD flow of an electrically conducting fluid with particular reference to blood flow in arteries in a slowly varying channel in the presence of a uniform transverse magnetic field was studied in [11]. A mathematical model of the flow of a bio magnetic viscoelastic fluid over a stretching sheet was developed in [12]. The flow and heat transfer of an HD viscoelastic fluid in a channel with stretching walls investigated in [13]. Misra and his research group made a great contribution to the field of physiological fluid dynamics. Radiation and free convection through a porous medium was studied in [14]. The effect of thermal radiation on an MHD flow of blood and heat transfer in a permeable capillary in stretching motion was been studied in [15]. A numerical model for a magneto hydrodynamic flow of blood in a porous channel was examined in [16]. A numerical study of flow and heat transfer during oscillatory blood flow in diseased arteries in the presence of magnetic fields was studied in [17]. The effect of an MHD free convection oscillatory Couette flow when the temperature and concentration oscillate with time in the presence of thermal radiation and chemical reaction was studied in [18]. The effect of thermal radiation, chemical reaction and viscous dissipation on free convection MHD flow was studied in [19]. Thermal radiation, chemical reaction, viscous and Joule dissipation effects on an MHD flow embedded in a porous medium was investigated in [20]. Mixed convection on an MHD flow with thermal radiation, chemical reaction and viscous dissipation embedded in a porous medium was examined in [21].

Even though the problem of unsteady magnetohydrodynamic flow with heat and mass transfer of blood in a narrow permeable blood vessel is significant, the effect of chemical reaction parameter on blood flow has not been considered [15]. This omission encourages considering thermal radiation and chemical reaction effects on an MHD flow of blood in a stretching permeable vessel.

The main objective of this paper is to study the effects of thermal radiation and chemical reaction on an MHD flow of blood in a stretching permeable vessel with no velocity, thermal and concentration slip at the vessel wall. 


\section{Mathematical formulation and analysis of the model}

Consider a blood flow in stretching permeable narrow vessels with the influence of a time dependent magnetic field which in two dimensional, unsteady, incompressible, thermal radiating, chemically reacting and electrically conducting. The $x y$ axes are taken in the direction parallel and perpendicular to the axes of the blood vessel.

The flow configuration of blood in the vessel and the coordinate system of the model are shown in Fig.1. At $y=0$, the temperature of blood at any point in the vessel is equal to the temperature of the blood vessel wall, the velocity of blood along the axis of the vessel is equal to the stretching velocity of blood, concentration of blood at any point is the same as blood concentration along the $x$ axis and the transverse velocity of blood in the vessel is equal to suction or injection velocity. As $y \rightarrow \infty$, the velocity of blood along the axis of the vessel is equal to zero, the temperature of blood at any point in the vessel is equal to the ambient temperature of the fluid and blood concentration along the $x$ axis is equal the ambient concentration of solute.

Consider a blood flow in the vessel which is influenced by a time dependent magnetic field of intensity which acts in the direction transverse to the blood. At time $t=0$, the blood vessel is assumed to be stretched with velocity $U_{w}(x, t)$ and $C_{w}(x, t)$ is blood concentration taken along the $x$ axis. The origin is kept fixed in the fluid medium of ambient temperature $T_{\infty}$ and ambient concentration of solute $C_{\infty}$.

Although according to Misra et al. [16] blood flow in the circulatory system is three dimensional, in many cases blood flow in a narrow vessel is taken to be two dimensional.

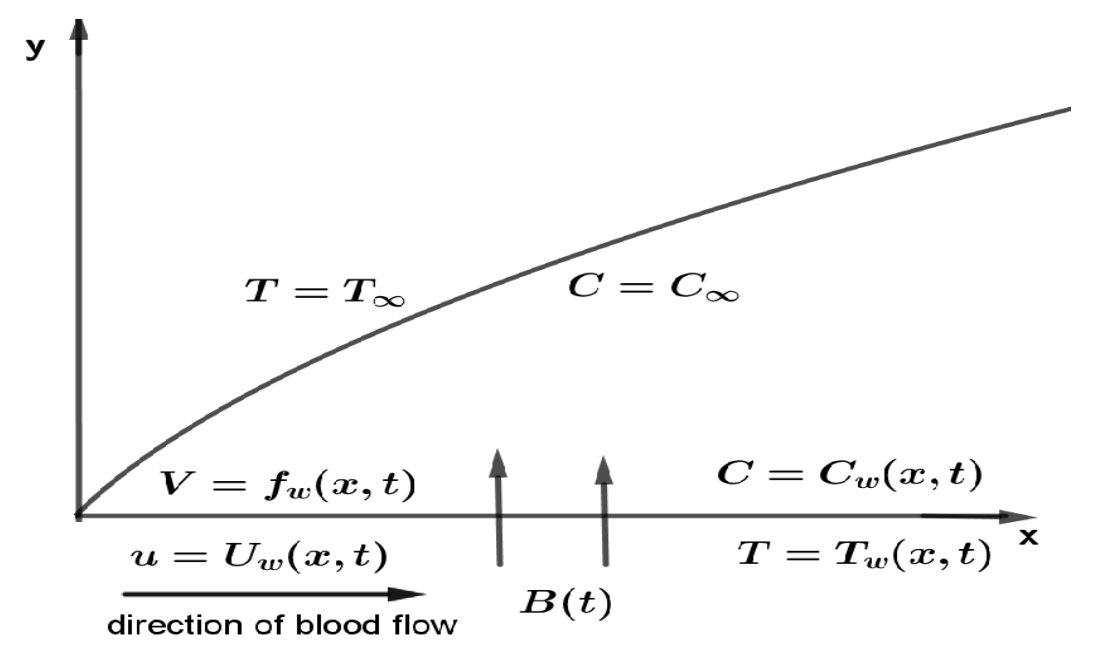

Fig.1. Flow configuration of blood in the vessel and coordinate system.

To derive the governing equations of the model the following assumptions are made:

1. Blood is an electrically conducting fluid hence the flow has magnetohydrodynamic property.

2. The magnetic field is time dependent which acts in the direction transverse to the flow of blood.

3. It is assumed that the magnetic Reynolds number is much less than unity so that the induced magnetic field is negligible in comparison to the applied magnetic field.

4. Velocity, thermal and concentration slip are negligible.

5. Blood flow through narrow vessels is taken as two dimensional.

6. The time dependent permeability parameter of blood vessel is taken into account.

7. Thermal radiation and chemical reaction that take place during blood flow in a narrow vessel are taken into consideration. 
Based on the model assumptions, the governing equations have the following form:

The continuity equation of the model has the form

$$
\frac{\partial u}{\partial x}+\frac{\partial v}{\partial y}=0
$$

In Eq.(2.1), $u$ denotes velocity of the blood flow along the $x$ axis of the vessel and $v$ represents transverse velocity of blood flow in the vessel.

The momentum equation of the model takes the form

$$
\frac{\partial u}{\partial t}+u \frac{\partial u}{\partial x}+v \frac{\partial u}{\partial y}=v \frac{\partial^{2} u}{\partial y^{2}}-\frac{\sigma B^{2}(t) u}{\rho}-\frac{v}{k_{l}(t)} u
$$

In Eq.(2.2), the second and third term on the left hand side represent convective acceleration. The second and third term on the right hand side denotes the time dependent magnetic field intensity due to Lorenz force and time dependent permeability parameter of blood, respectively.

The energy equation of the model can be expressed as

$$
\frac{\partial T}{\partial t}+u \frac{\partial T}{\partial x}+v \frac{\partial T}{\partial y}=\alpha \frac{\partial^{2} T}{\partial y^{2}}-\frac{1}{\rho C_{p}} \frac{\partial q_{r}}{\partial y} .
$$

In Eq.(2.3), $\alpha$ denotes thermal diffusivity, $\rho$ represents density of blood.

The concentration equation of the model takes the form

$$
\frac{\partial C}{\partial t}+u \frac{\partial C}{\partial x}+v \frac{\partial C}{\partial y}=D \frac{\partial^{2} C}{\partial y^{2}}-k_{r}\left(C-C_{\infty}\right) .
$$

In Eq.(2.4), $D$ denotes molecular diffusivity and $k_{r}$ represents the chemical reaction parameter. The boundary conditions for the problem can be written as

$$
\begin{aligned}
& u=U_{w}, \quad v=f_{w}, \quad T=T_{w}, \quad C=C_{w} \text { at } \quad y=0, \\
& u \rightarrow 0, \quad T \rightarrow T_{\infty}, \quad C \rightarrow C_{\infty} \text { as } \quad y \rightarrow \infty .
\end{aligned}
$$

In Eqs (2.6), $f_{w}$ denotes the blood velocity at the vessel wall and is equal to injection/suction velocity and given by

$$
f_{w}=-\left(\frac{v V_{w}}{x}\right)^{1 / 2} S
$$

In Eq.(2.7), the concentration transfer of blood at the surface of the vessel wall takes place with a velocity $f_{w}$, where $f_{w}>0$ in case of injection and $f_{w}<0$ in case of suction. 
In Eq.(2.2), $k_{l}(t)=k_{2}(1-c t)$ represents the time dependent permeability parameter. The velocity $U_{w}(x, t)$ is the stretching velocity of the blood vessel; the surface temperature is $T_{w}(x, t)$; the concentration of blood is $C_{w}(x, t)$ and time dependent chemical reaction parameter $k_{r}(t)$ takes the following form

$$
\begin{aligned}
& U_{w}(x, t)=\frac{a x}{1-c t}, T_{w}(x, t)=T_{\infty}+\frac{b x}{1-c t}, C_{w}(x, t)=C_{\infty}+\frac{b x}{1-c t}, k_{r}(t)=\frac{k_{0}}{1-c t} \\
& k_{r}(t)=\frac{k_{0}}{D(1-c t)}
\end{aligned}
$$

where $a, b, c$ and $k_{0}$ are the constants such that $a>0, b \geq 0, c \geq 0$ and $c t<1$. Let us choose $B(t)=\frac{B_{0}}{\sqrt{(1-c t)}}, B_{0}$ denotes the magnetic field strength at $t=0$ and $K_{r}(t)$ is chemical reaction rate of the blood flow.

The Roseland approximation for radiative heat flux [Brewster, 1992] is given by

$$
q_{r}=\left(\frac{-4 \sigma^{*}}{3 k_{s}}\right)\left(\frac{\partial T^{4}}{\partial y}\right) .
$$

In Eq.(2.9), the parameters $\sigma^{*}$ and $k_{s}$ represent the Stefan Boltzmann constant and the Roseland mean absorption coefficient, respectively.

We now assume that the temperature difference of the blood flow inside the vessel is sufficiently small, $T^{4}$ in Eq.(2.10) can be expanded as a linear function of $T_{\infty}$, using the Taylor series expansion and neglecting higher-order terms we write $T^{4}$ as

$$
T^{4} \cong 4 T_{\infty}^{3} T-3 T_{\infty}{ }^{4}
$$

In Eq.(2.10), $T_{\infty}$ denotes the embedded temperature.

\section{Non dimensionalization of the model} quantities

Dimensionless form of the model problem is obtained by introducing the following non dimensional

$$
\eta(x, y, t)=y \sqrt{\frac{U_{w}}{v x}}, \quad \psi(x, y, t)=\sqrt{v x U_{w}} f(\eta), \quad \theta(\eta)=\frac{T-T_{\infty}}{T_{w}-T_{\infty}}, \quad \phi(\eta)=\frac{C-C_{\infty}}{C_{w}-C_{\infty}} .
$$

In Eq.(3.1) $f, \eta, \psi, \theta$ and $\phi$ denote the dimensionless stream function, similarity variable, stream function, dimensionless temperature and dimensionless concentration, respectively. And prime denotes derivatives with respect to $\eta$.

Where $\psi(x, y, t)$ is the stream function defined by $u=\frac{\partial \psi}{\partial y}$ and $v=-\frac{\partial \psi}{\partial x}$ which satisfies continuity Eq.(2.1).

Substitute Eq.(3.1) into Eqs (2.2)-(2.4) the following non dimensional equations are obtained 


$$
\begin{aligned}
& f^{\prime \prime \prime}+f f^{\prime \prime}-f^{\prime 2}-A\left(f^{\prime}+\frac{\eta}{2} f^{\prime \prime}\right)-M^{2} f^{\prime}-\frac{1}{k_{3}} f^{\prime}=0, \\
& \left(\frac{1+R}{\operatorname{Pr}}\right) \theta^{\prime \prime}+f \theta^{\prime}-\theta f^{\prime}-A\left(\theta+\frac{\eta}{2} \theta^{\prime}\right)=0, \\
& \phi^{\prime \prime}+\operatorname{Sc}\left(f \phi^{\prime}-f^{\prime} \phi\right)-A \operatorname{Sc}\left(\frac{1}{2} \eta \phi^{\prime}+\phi\right)-K_{r} \phi=0
\end{aligned}
$$

where $A$ is the unsteadiness parameter; $M$ is the Hartmann number; $R$ is the radiation parameter; $k_{3}$ is the permeability parameter and $\operatorname{Pr}$ is the Prandtl number.

Here

$$
A=c / a ; a=v(1-c t) ; \quad M=B_{0} \sqrt{\sigma / \rho a} ; R=16 \sigma^{*} T_{\infty}^{3} / 3 k_{s} k ; \kappa_{3}=a \kappa_{2} / v ; \mathrm{Sc}=v / D \text { and } \operatorname{Pr}=v / \alpha
$$

The boundary conditions corresponding to Eqs (2.5)-(2.6) are given by

$$
\begin{aligned}
& f(0)=S, f^{\prime}(0)=1, \theta(0)=1, \\
& f^{\prime}(\eta) \rightarrow 0, \theta(\eta) \rightarrow 0, \phi(\eta) \rightarrow 0 \quad \text { as } \quad \eta \rightarrow \infty
\end{aligned}
$$

In Eqs (3.5), $S>0$ and $S<0$ denote suction and injection parameters, respectively.

The non linear partial differential Eqs (3.2)-(3.4) subjected to the boundary conditions (3.5)-(3.6) constitute the non dimensional form of the present model.

\section{Numerical solution of the model problem}

The set of coupled non-linear Eqs (3.2)-(3.4) is solved numerically using ode45 MATLAB code. First, convert the higher order non linear differential equations to a system of first order ordinary differential equations by using

$$
\begin{aligned}
& y_{1}=f, \quad y_{2}=f^{\prime}, \quad y_{3}=f^{\prime \prime}, \quad y_{4}=\theta, \quad y_{5}=\theta^{\prime}, \quad y_{6}=\phi, \quad y_{7}=\phi^{\prime}, \\
& y_{1}^{\prime}=y_{2}, \\
& y_{2}^{\prime}=y_{3}, \\
& y_{3}^{\prime}=y_{2}^{2}-y_{1} y_{3}+A\left(y_{2}+\eta / 2 y_{3}\right)+y_{2}\left(M^{2}+1 / k_{3}\right), \\
& y_{4}^{\prime}=y_{5}, \\
& y_{5}^{\prime}=\operatorname{Pr} /(1+R)\left[y_{1} y_{5}+y_{2} y_{4}+A\left(y_{4}+\eta / 2 y_{5}\right)\right],
\end{aligned}
$$




$$
\begin{aligned}
& y_{6}^{\prime}=y_{7}, \\
& y_{7}^{\prime}=-\operatorname{Sc}\left(y_{1} y_{7}-y_{2} y_{6}\right)+A \operatorname{Sc}\left(\eta / 2 y_{7}+y_{6}\right)-k_{r} y_{6} \\
& y_{7}^{\prime}=-\operatorname{Sc}\left(y_{1} y_{7}-y_{2} y_{6}\right)+A \operatorname{Sc}\left(\eta / 2 y_{7}+y_{6}\right)+k_{r} y_{6} .
\end{aligned}
$$

With corresponding boundary conditions

$$
\begin{aligned}
& y_{1}(\eta)=S, \quad y_{2}(\eta)=1, \quad y_{4}(\eta)=1, \quad y_{6}(\eta)=1, \quad y_{3}(\eta)=m \\
& y_{5}(\eta)=n, \quad y_{7}(\eta)=l
\end{aligned}
$$

with corresponding boundary conditions

$$
\begin{aligned}
& y_{1}(0)=S, \quad y_{2}(0)=1, \quad y_{4}(0)=1, \quad y_{6}(0)=1, \quad y_{3}(0)=m, \\
& y_{5}(0)=n, \quad y_{7}(0)=l
\end{aligned}
$$

where $m, n$ and $l$ are unknown to be determined as part of numerical solution using ode 45 .

\section{Simulation study}

The effect of thermal radiation and chemical reaction on the MHD flow of blood in a stretching permeable vessel with the influence of a time dependent magnetic field intensity has been studied. The effects of physical parameters, viz., the permeability parameter, Hartmann number, unsteadiness parameter, thermal radiation parameter, chemical reaction parameter and Schmidt number on flow variables, viz., the velocity, temperature and concentration of blood flow have analyzed. The governing non-linear partial differential equations of unsteady blood flow, temperature and concentration profiles are transformed to a set of ordinary differential equations using similarity transformations which can be solved numerically using ode45 MATLAB code.

For the sake of brevity only graphical representations of temperature profiles for different values of the thermal radiation parameter, Prandtl number and Hartmann number; velocity profiles for different values of the permeability parameter and unsteadiness parameter. Furthermore, concentration profiles for different values of the time dependent chemical reaction parameter and Schmidt number are presented. 


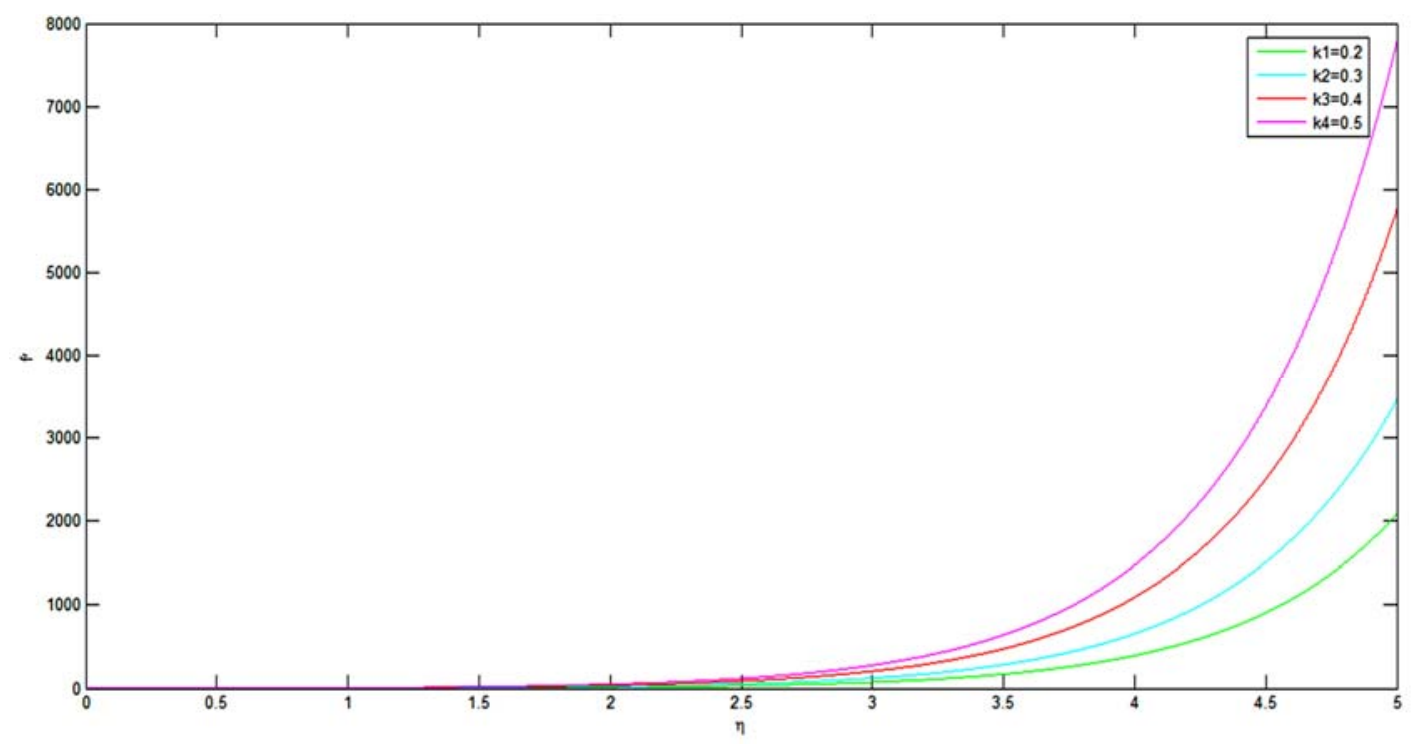

Fig.2. Velocity profile of the model for different values of the permeability parameter.

Figure 2 shows the velocity profile of blood flow for different values of the permeability parameter. The graph shows $f^{\prime}$ versus $\eta$ for different values of dimensionless velocity against dimensionless distance. As the values of the permeability parameter increase, the velocity profile of the blood flow increases.

For a particular value of the permeability parameter as the dimensionless distance increases the velocity of the blood flow increases and attains its maximum value. Physiologically, this shows the relationship between velocity profiles of the blood flow and the permeability parameter of a porous medium i.e., the volume of blood that passes through the porous medium dominates the total volume. Hence, the fluid particles move from one place to another place easily in the vessel.

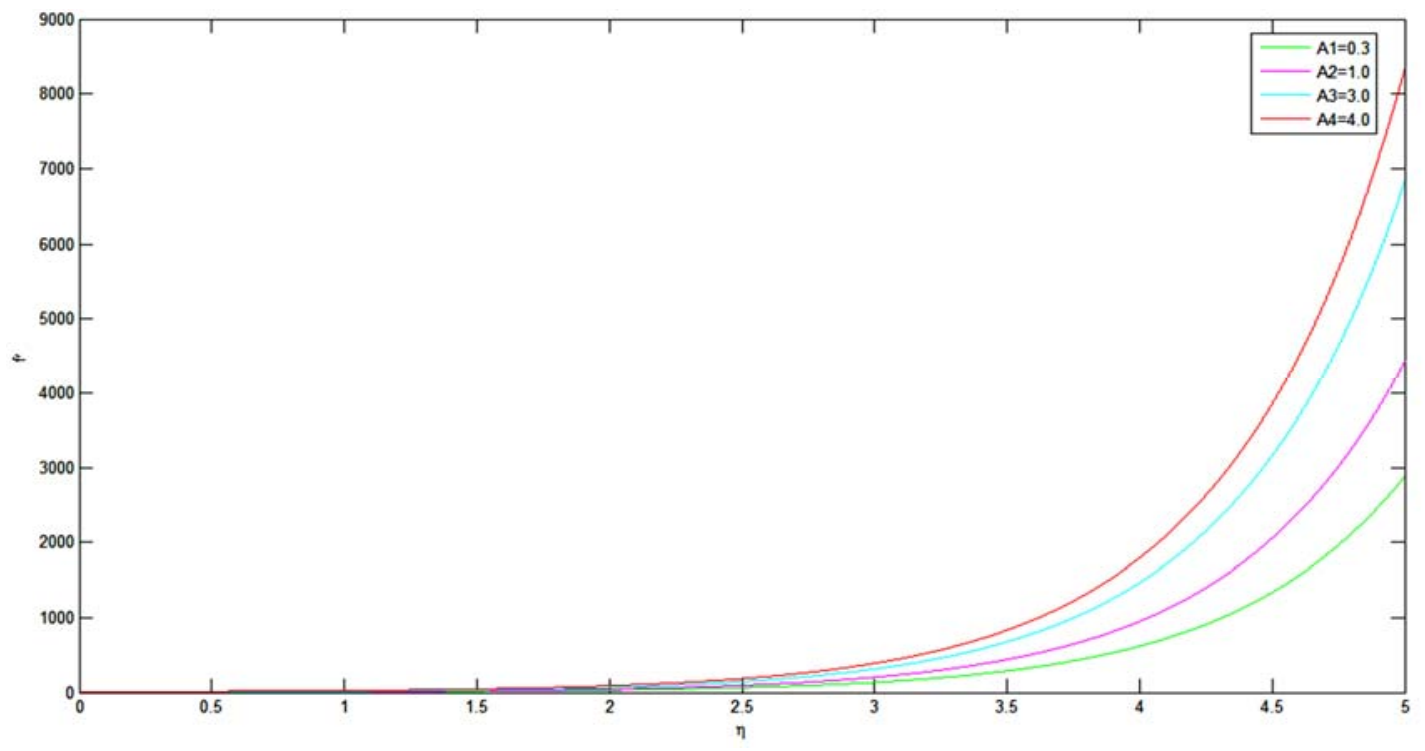

Fig.3. Velocity profile of the model for different values of unsteadiness parameter.

Figure 3 shows the effect of the unsteadiness parameter on the velocity of blood flow. The graph is plotted for $f^{\prime}$ versus $\eta$ with dimensionless velocity and dimensionless distance, respectively. The simulation study shows that increasing values of the unsteadiness parameter result in an increment in the velocity of blood flow in the vessel with no slip at the boundary. 


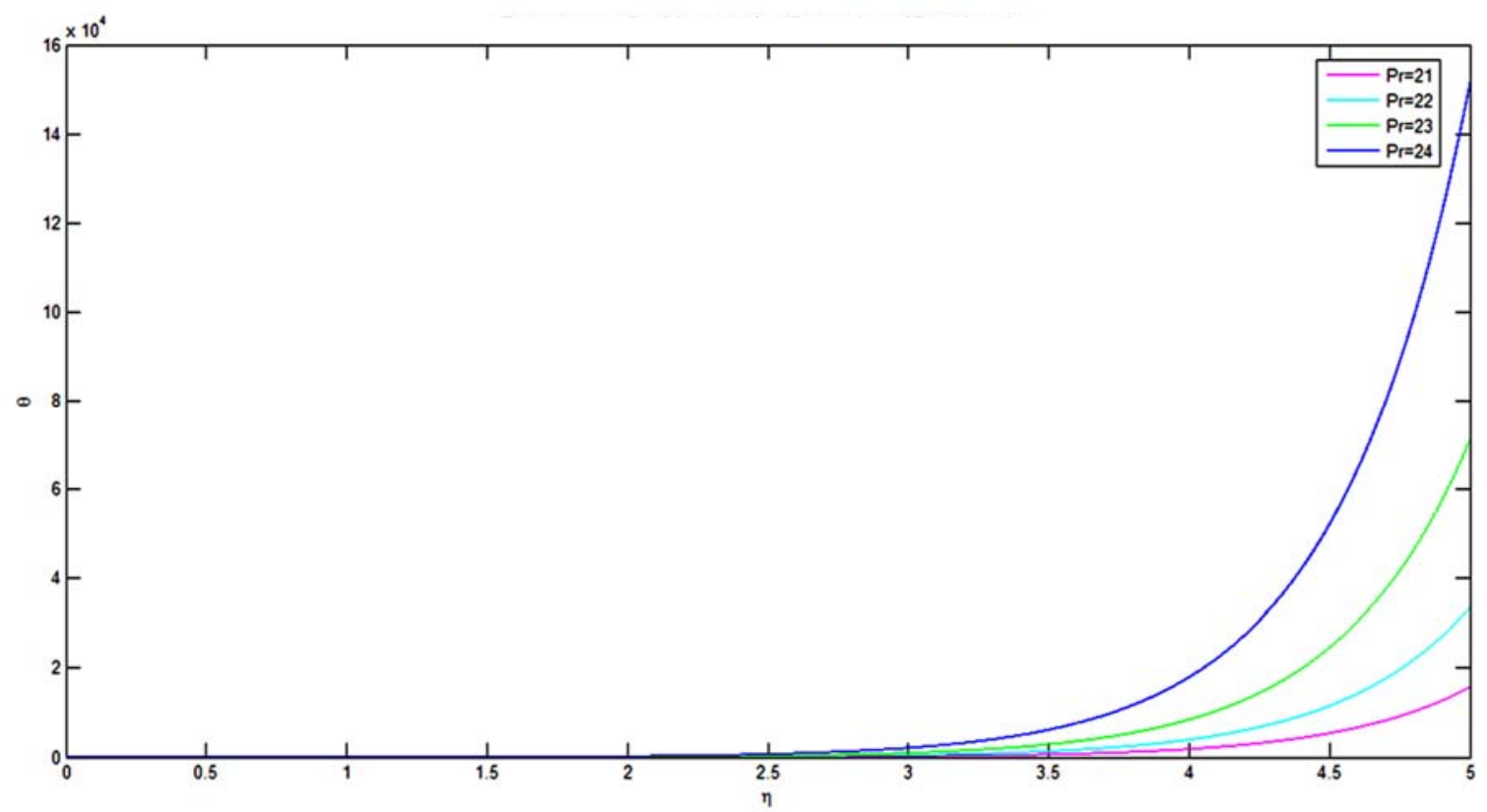

Fig.4. Temperature profile of the model for different values of Prandtl number.

Figure 4 presents the change in the temperature profile for different values of the Prandtl number. The graph is plotted for $\theta$ versus $\eta$ which represents dimensionless temperature in the vertical axis and dimensionless distance in the horizontal axis respectively. This figure shows that the temperature gradient at the wall increases with an increment of the Prandtl number. Physiologically, this can be interpreted as an increment of the Prandtl number is accompanied by an enhancement of heat transfer at the wall of the blood vessel. The achievement of a higher Prandtl number in the blood results in a decrement of thermal conductivity and a result heat conduction capacity decreases. Consequently, the thermal boundary layer thickness decreases, hence the heat transfer rate at the wall of the blood vessel increases.

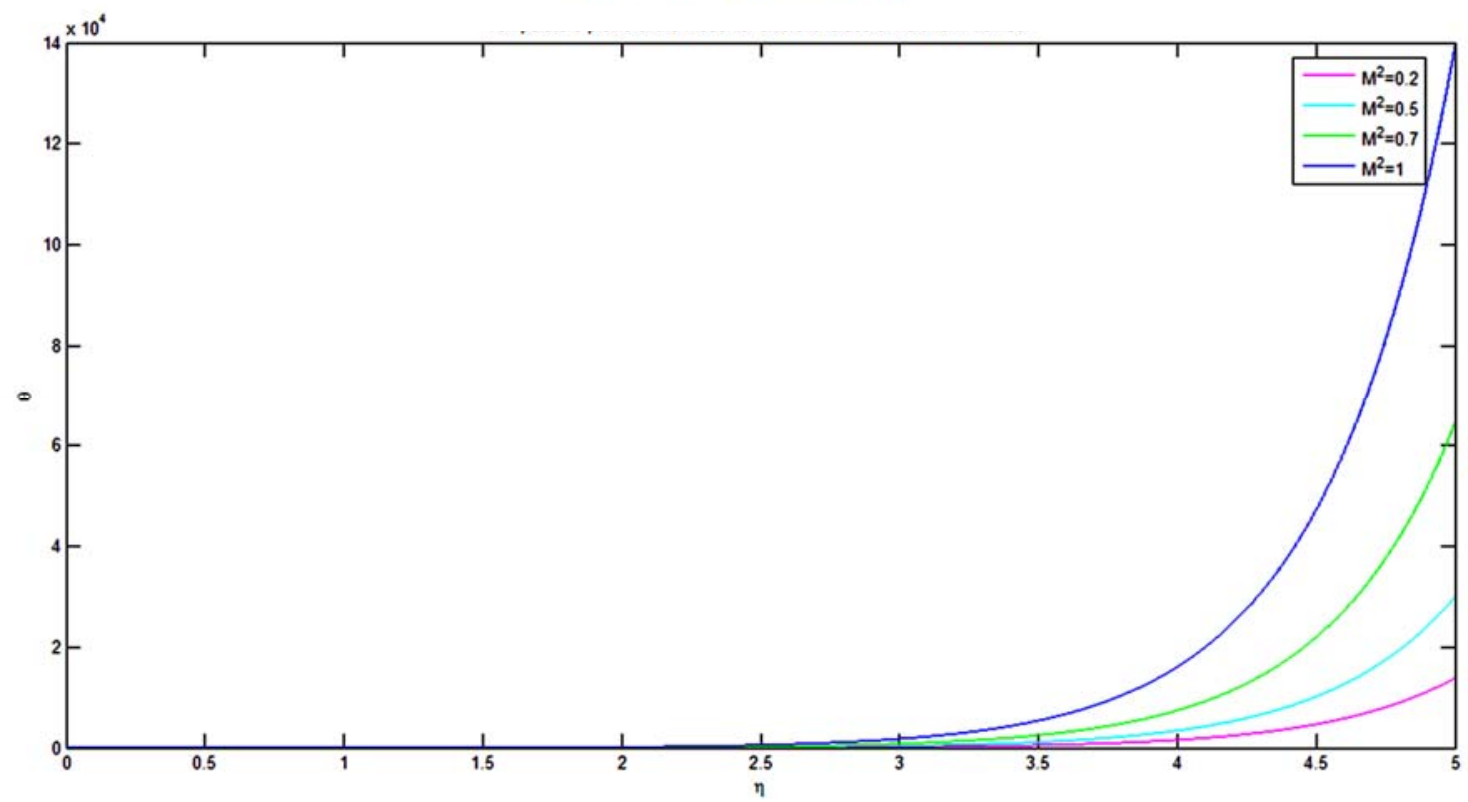

Fig.5. Temperature profile of the model for different values of the Hartmann number. 
Figure 5 shows characteristics of the temperature profile for different values of the Hartmann number. The graph is plotted for $\theta$ versus $\eta$ along vertical and horizontal axes, respectively. From the simulated graph it can be concluded that as the Hartmann number increases the temperature increases. Physiologically, this means blood behaves as an electrically conducting fluid which induces electric and magnetic field. Both electric and magnetic field enhance the temperature of the boundary layer of the blood vessel. The combined effect of both forces produces Lorentz force which increases the temperature of blood.

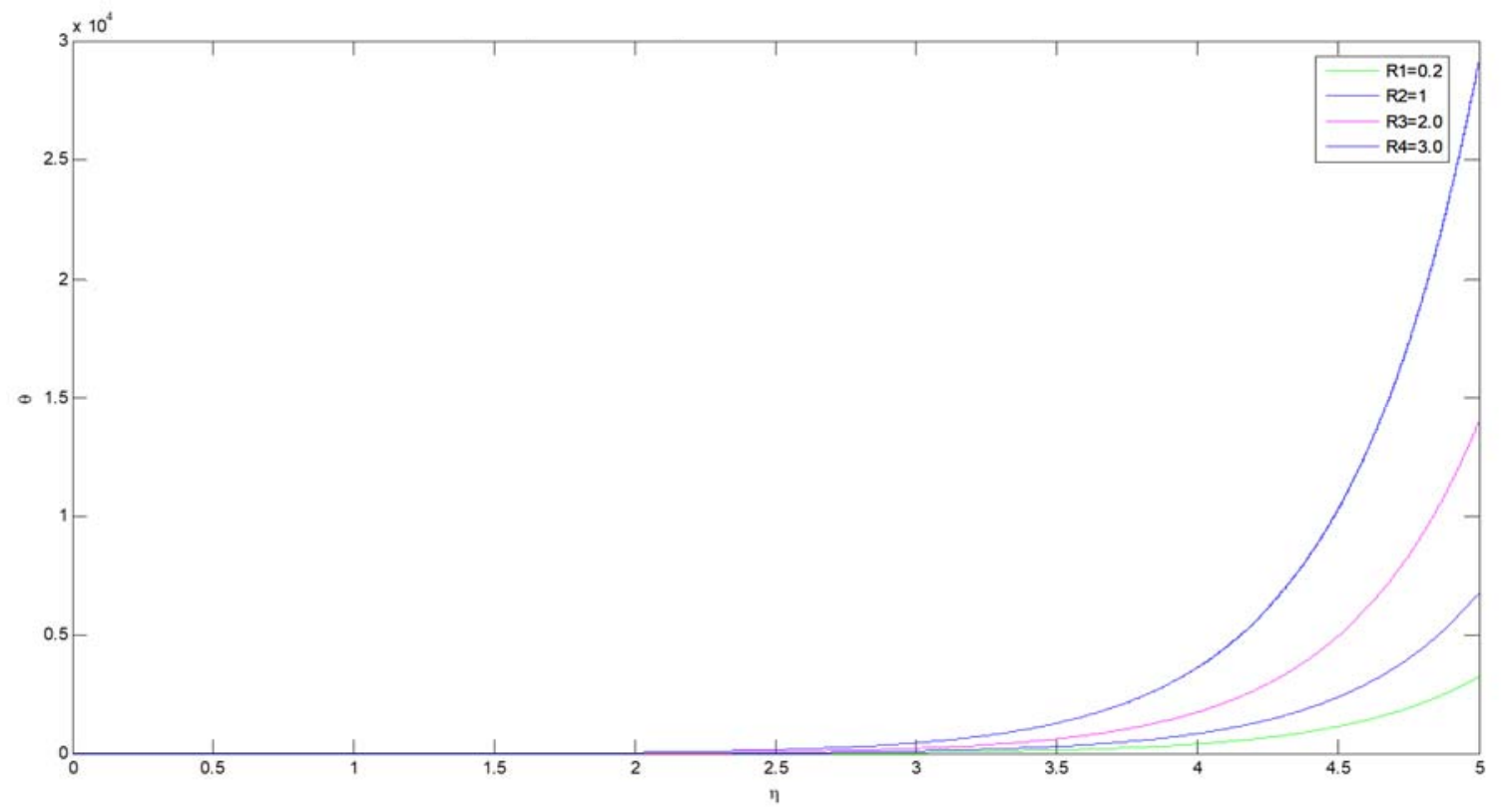

Fig.6.Temperature profile of the model for different values of thermal radiation parameter.

Figure 6the effect of thermal radiation on the temperature profile has been presented. The graph is plotted for $\theta$ versus $\eta$ for different values of dimensionless temperature against dimensionless distance. The result of the simulation study shows that as the thermal radiation increases during blood flow in the vessel there is a significant rise in the thickness of the boundary layer. Hence the temperature of the boundary layer increases which results in an increment of temperature.

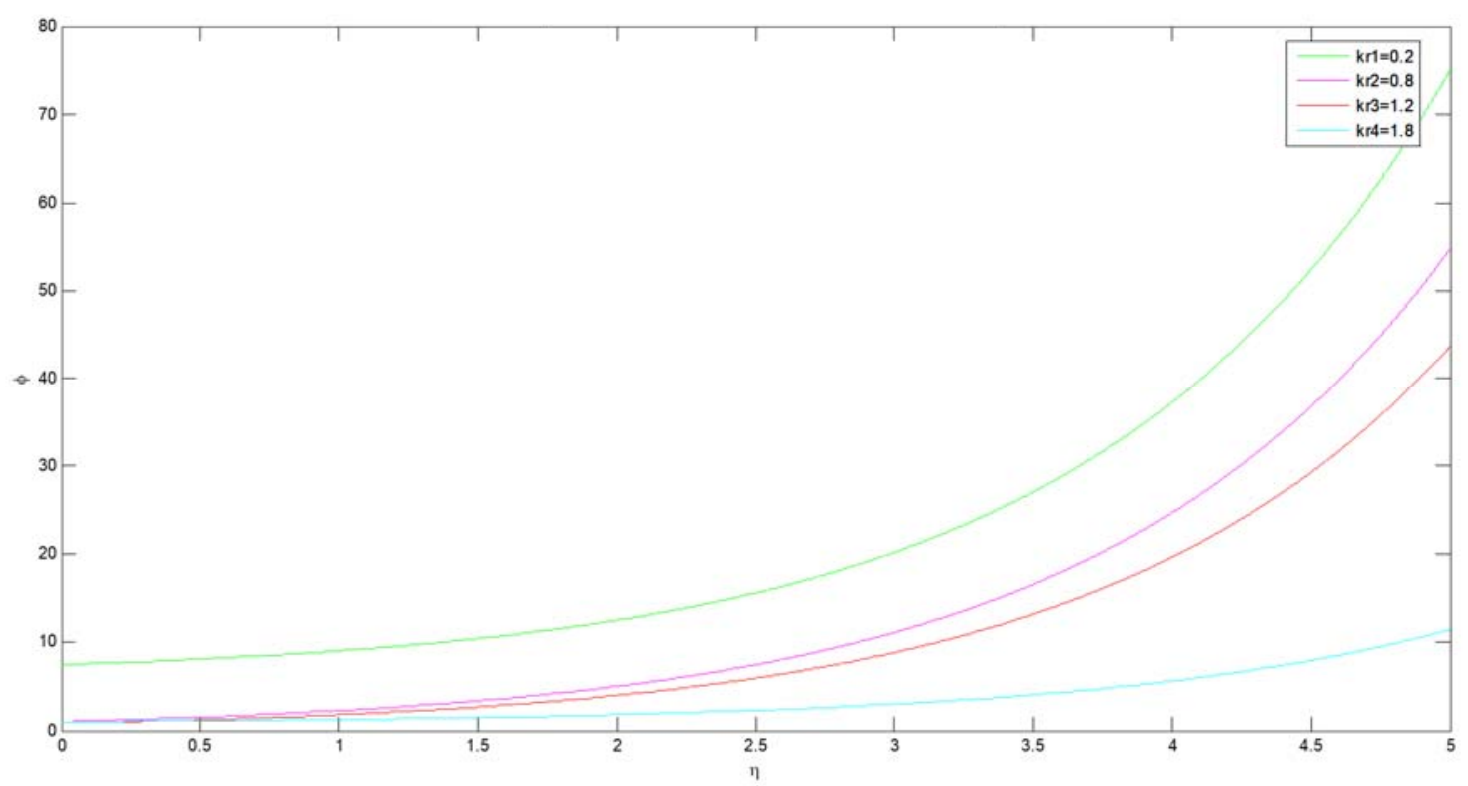

Fig.7. Concentration profile of the blood flow for different values of the chemical reaction parameter. 
Figure 7 shows the effect of the chemical reaction parameter on the concentration profile of blood. The graph shows $\phi$ versus $\eta$ representing, respectively, dimensionless concentration and distance. From the simulation study it can be observed that as the chemical reaction parameter increases the concentration of blood flow decreases. Physiologically, this shows that higher values of the chemical reaction parameter result in a decrement of molecular diffusivity which directly decreases the concentration of blood.

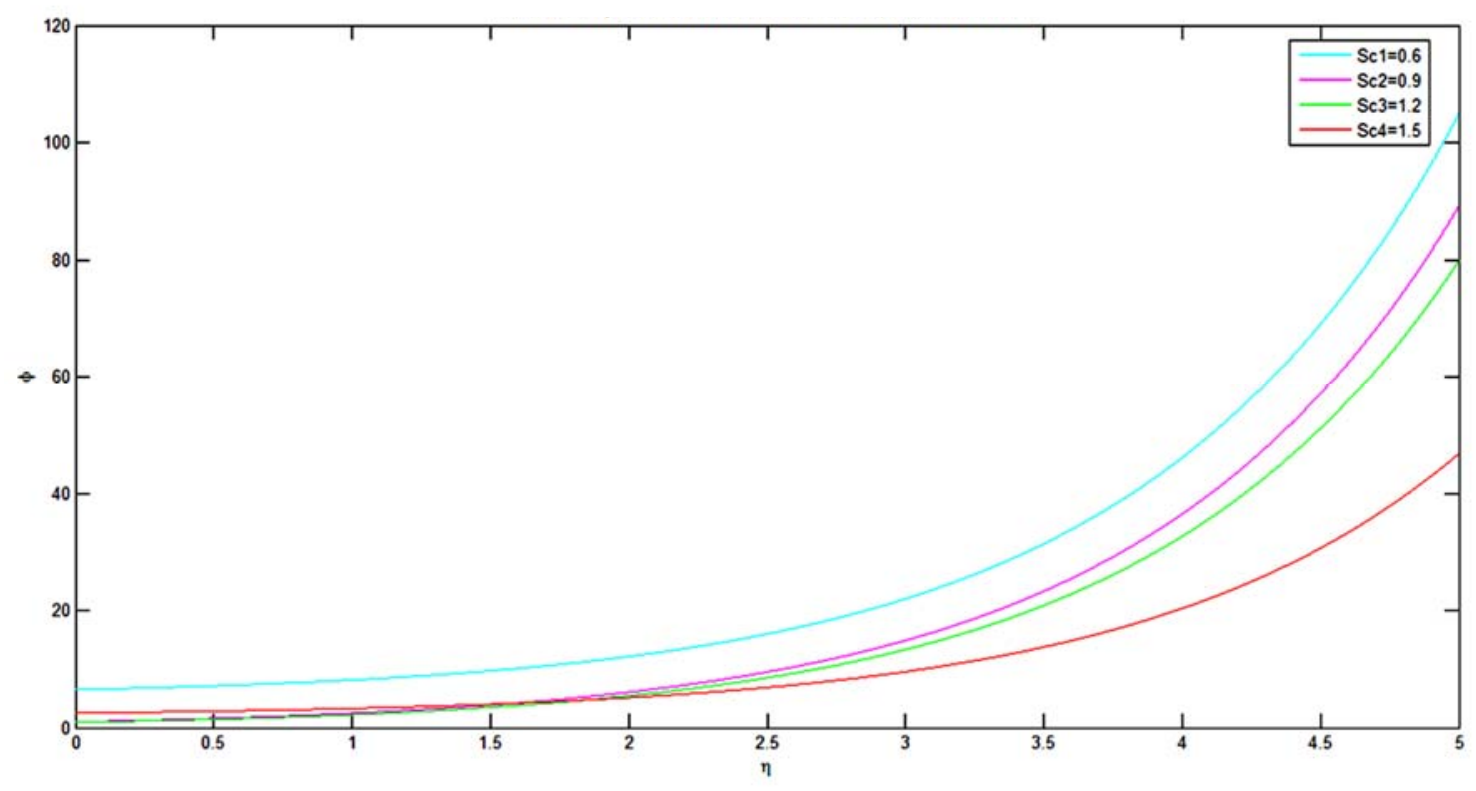

Fig. 8. Concentration profile of the model for different values of the Schmidt number.

Figure 8 shows the effect of the Schmidt number on the concentration profile of blood. The graph shows $\phi$ versus $\eta$ representing respectively, dimensionless concentration and distance. From the simulation study it can be observed that as the Schmidt number increases the concentration of blood flow decreases. Physiologically, this shows blood can diffuse slowly in the vessel.

\section{Theoretical estimates and experimental results}

The mass and heat transfer of time dependent stretching blood flow in a permeable blood vessel influenced by the action of time dependent external magnetic field was studied. A numerical method can be applied to the system of Eqs (3.2) -(3.4) with the corresponding boundary conditions Eqs (3.5)-(3.6).

To apply the numerical method it is important to assign some values for the dimensionless parameters: the suction parameter, unsteadiness parameter, permeability parameter, Hartmann number and radiation parameter from theoretical and experimental studies. As mentioned in the experimental study of Valvano et al. (1994) and Chato (1980) at temperature $310^{\circ} \mathrm{k}$ the human blood has the following data for dynamic viscosity $\mu=3200 \frac{\mathrm{kg}}{\mathrm{ms}}$, the specific heat capacity at constant pressure $C_{p}=14.65 \frac{\mathrm{J}}{\mathrm{kg} 0_{\mathrm{k}}}$ and thermal conductivity $2.2 \times 10^{-3} \frac{\mathrm{J}}{\mathrm{ms} \mathrm{O}_{k}}$. Using these values the Prandtl number of human blood is 21 .

The numerical results of the present study are compared with Ishak et al. (2009) and Salem and ElAziz (2008) in order to check the validity of the present work. The results are in good agreement with the above mentioned works. 


\section{Conclusion}

In this paper, blood flow in the presence of thermal radiation and chemical reaction under the influence of time dependent magnetic field intensity has been analysed theoretically. The non-linear partial differential equations are converted to ordinary differential equations using similarity transformations and solved numerically.

From the simulation study the following important results are obtained:

1. Velocity of the blood flow increases with an increment of both permeability and unsteadiness parameter.

2. Temperature of the blood increases at the vessel wall as the Prandtl number and Hartmann number increase.

3. Concentration of the blood decreases as the time dependent chemical reaction parameter and Schmidt number increase.

\section{Application of the model problem}

The present study can be applied to model blood flow in micro vessels

1. To help patients in the treatment of pulmonary thrombus embolism (PTE).

2. It can also be applied in the treatment of blood cancer and tumor using electromagnetic radiation.

3. Infrared radiation can be applied directly in the treatment of blood cancer by heat therapy to treat the infected areas of the blood.

4. This study helps radiologist to find applications in estimating how to reduce electromagnetic radiation.

5. This study will be helpful in evaluating the accuracy of future theoretical works.

\section{Nomenclature}

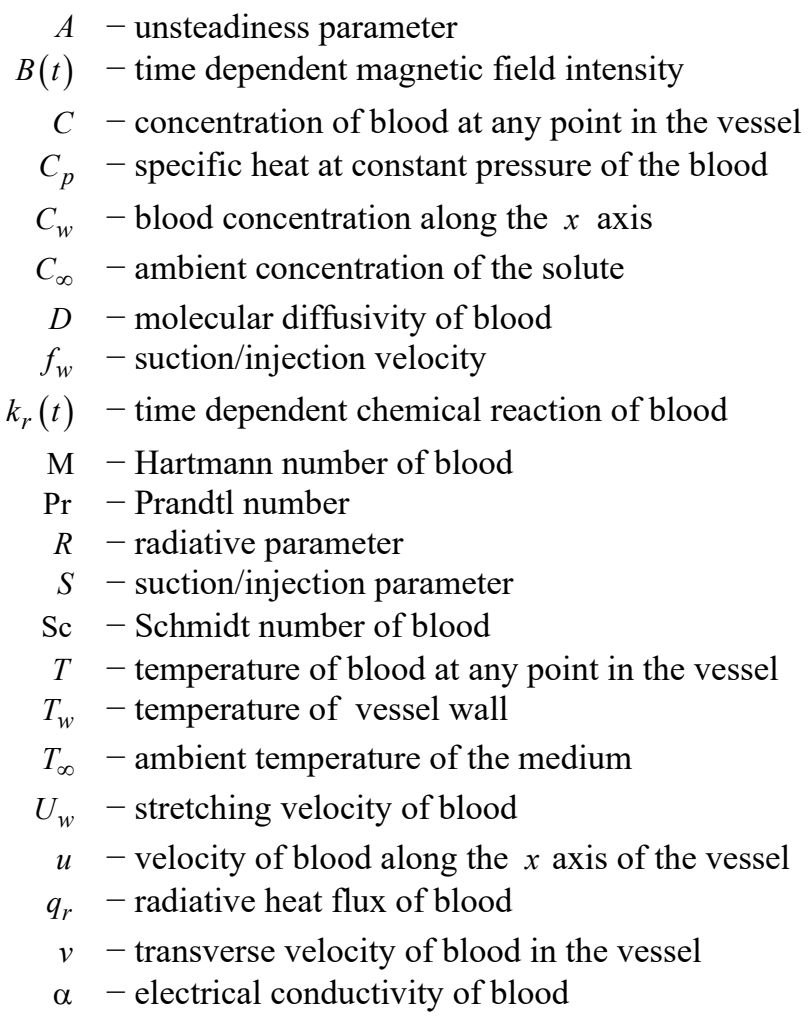




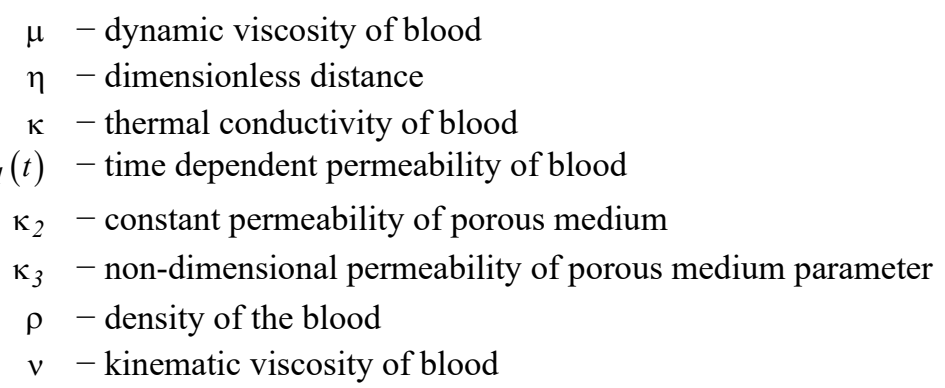

\section{References}

[1] Prakash J. and Makinde O.D. (2011): Radiative heat transfer to blood flow through a stenotic artery in the presence of magnetic field. - Lat. Am. Appl. Res., vol.41, pp.273-277.

[2] He Y., Shirazaki M., Liu H., Himeno R. and Sun Z. (2006): A numerical coupling model to analyze the blood flow, temperature, and oxygen transport in human breast tumor under laser irradiation. - Comp. Biol. Med., vol.36, pp.1336-1350.

[3] Ogulu A. and Bestman A.R. (1994): Blood flow in a curved pipe with radiative heat transfer. - Acta Physica Hungarica, vol.74, pp.189-201.

[4] Misra J.C., Sinha A. and Shit G.C. (2010): Flow of a biomagnetic viscoelastic fluid: application to estimation of blood flow in arteries during electromagnetic hyperthermia, a therapeutic procedure for cancer treatment. Appl. Math. Mech. Eng. Educ., vol.31, pp.1405-1420.

[5] Thomas Hernandez et al. Alligator (1983): Metabolism studies on chemical reactions in vivo. - Comparative Biochemistry and Physiology Part B: Comparative Biochemistry, vol.74, No.1, pp.1-175.

[6] Zhiliang Xu, Nan Chen, Shawn C. Shadden, Jerrold E. Marsden, Malgorzata M. Kamocka, Elliot D. Rosen and Mark Alber (2009): Study of blood flow impact on growth of thrombi using a multiscale model. - Soft Matter, vol.5, No.4, pp.769-779.

[7] Madhu Sharma and Gaur R.K. (2017): Effect of variable viscosity on chemically reacting magneto-blood flow with heat and mass transfer. - Global Journal of Pure and Applied Mathematics, vol.13, No.3, pp.26-35.

[8] Vankan W.J., Huyghe J.M., Drost M.R., Janssen J.D. and Huson A. (1997): A finite element mixture model for hierarchical porous media. - Int. J. Numer. Math. Eng., vol.40, pp.197-210.

[9] Dash R.K., Mehta K.N. and Jayaraman G. (1996): Casson fluid flow in a pipe filled with homogeneous porous medium.- Int. J. Eng. Sci.,vol.34, pp.1146-1156.

[10] Preziosi L. and Farina A. (2002): On Darcy's law for growing porous media. - Int. J. Non-linear Mech., vol.37, pp.485-491.

[11] Pal B., Misra J.C. and Gupta A.S. (1996): Steady hydromagnetic flow in a slowly varying channel. - Proc. Natl. Inst. Sci. Ind. Part A,vol.66, pp.247-262.

[12] Misra J.C. and Shit G.C. (2009): Biomagnetic viscoelastic fluid flow over a stretching sheet. - Appl. Math. Comput., vol.210, pp.350-361.

[13] Misra J.C., Shit G.C. and Rath H.J. (2008): Flow and heat transfer of a MHD viscoelastic fluid in a channel with stretching walls: some applications to hemodynamics. - Comput. Fluids, vol.37, pp.1-11.

[14] Raptis A. (1998): Radiation and free convection flow through a porous medium. - Int. Commun. Heat Mass,vol.25, pp.289-295.

[15] Misra J.C. and Sinha A. (2013): Effect of thermal radiation on MHD flow of blood and heat transfer in a permeable capillary in stretching motion. - Heat Mass Transfer, vol.49, pp.617-628.DOI 10.1007/s00231-0121107-6. 
[16] Misra J.C., Sinha A. and Shit G.C. (2011): A numerical model for magnetohydrodynamic flow of blood in a porous channel. - J. Mech. Biol.,vol.11, pp.547-562.

[17] Sinha A. and Misra J.C. (2012): Numerical study of flow and heat transfer during oscillatory blood flow in diseased arteries in presence of magnetic fields. - Appl. Math. Mech. Engl. Educ., vol.33, pp.649-662.

[18] Zigta B. and Koya P.R. (2017): The effect of MHD on free convection with periodic temperature and concentration in the presence of thermal radiation and chemical reaction. - International Journal of Applied Mechanics and Engineering, vol.22, No.4, pp.1059-1073.DOI: 10.1515/ijame-2017-0068.

[19] Zigta B. (2018): The effect of thermal radiation, chemical reaction and viscous dissipation on MHD flow.International Journal of Applied Mechanics and Engineering, vol.23, No.3, pp.787-801. DOI: 10.2478/ijame2018-0043.

[20] Zigta B. (2019): Thermal radiation, chemical reaction, viscous and joule dissipation effects on MHD flow embedded in a porous medium. - International Journal of Applied Mechanics and Engineering, vol.24, No.3, pp.725-737. DOI: 10.2478/ijame-2019-0045.

[21] Zigta B. (2020): Mixed convection on MHD flow with thermal radiation, chemical reaction and viscous dissipation embedded in a porous medium. - International Journal of Applied Mechanics and Engineering, vol.25, No.1, pp.219-235. DOI: 10.2478/ijame-2020-0014.

Received: April 18, 2020

Revised: June 27, 2020 